\title{
Using Dental Parts as A Bone Graft, Smart Dentine Grinder, Kometa Bio
}

\section{Vicente Carrasco*}

Department of Dentistry, Mexico

*Corresponding Author: Vicente Carrasco Gutiérrez de Lara, Department of Dentistry, Mexico.

Received: October 28, 2019; Published: November 19, 2019

DOI: $10.31080 /$ ASDS.2019.03.0704

\begin{abstract}
Introduction: An extracted dental piece is no longer an organic waste product, it is crushed and conditioned in such a way that it is used as an autologous graft.

Objective: To present some cases of alveolar preservation in cases of surgical extraction of third molars as graft material for vertical increase of the alveolar process to receive oral implants.

Material and Methods: Review of clinical cases.

Results: Under the scanning electron microscope the Auto BT shows a consistency similar to that of the cortical bone, so that the use of teeth as an autogenous graft can be considered because they have physicochemical characteristics similar to autogenous bone grafts, what is demonstrated in the clinical cases that are exposed in the present work.

Conclusion: Auto BT can be used safely within a variety of reconstruction procedures such as maxillary sinus graft, guided bone regeneration, alveolar flange augmentation and post-extraction alveolus graft, which represents a good resource since autogenous tooth grafting it is safer than allogenous and xenogeneic grafts.
\end{abstract}

Keywords: Dental Pieces; Bone Graft

Dental extraction is one of the procedures that are mostly performed in the dental consultation, both privately and publicly. It is known that it is the therapeutic intended to remove the dental parts from its socket, in this panorama there are enough statistics on the reabsorption of the alveolar bone, which prove its consequence of a deformity that can be an obstacle to the conservation of aesthetics, phonetics and functionality.

An extraction affects the external cortical within a span of up to eight weeks and results in rapid and significant changes in the alveolar edge, also in the gingival contour and causes, as a consequence, an aesthetic deficiency and even the phonetic, which is why that the alveoli must be preserved, among other situations why it becomes very difficult to restore the natural dimensions, it should be considered that for a prosthetic restoration you have to have an adequate volume in all three dimensions, including cortical intact.

When a tooth extraction is performed, a loss of bone volume is caused however if the part is used as a graft to preserve the socket, the remodeling, which naturally exists, will be minor and the dimensional changes will not alter the volume of the alveolar bead, which is very useful for implant placement.

The dental piece as an autogenous graft, in the United States more than twenty million dental extractions are carried out per year, routinely the parts extracted were considered a clinical waste, however, they can be used for the creation of a high quality autogenous graft, due to the great potential as a bone inducer. 
Based on clinical studies on dental anchyloses, a system has been developed that allows obtaining from the newly removed dental part, a pathogen-free particulate graft for immediate use. The great advantage is that it can be used immediately to extraction, serves to preserve the size of the socket and for some constant situations in the daily clinic.

Bone graft materials Allogeneic bone and synthetic materials are important sources for grafting. Autogenous grafting is considered to be the "gold standard", as it exhibits a matrix of bioactive, nonimmunological or pathological instruction alcing cells, rather than the need to collect bone with the resulting morbidity.

The mandibular bone, alveolar and tooth development originate from neural crest cells and many proteins are common to bone, dentin and cement, Donovan., et al. 1993. Dentin and ankylosed cement are known to undergo a very slow remodeling by osteoclasts and then replaced with bone over time.

Schultz., et al. (2005) found development factors intact and preserved in an extracellular colagen matrix in ancient human bones and teeth.

Recently several studies have reported that extracted teeth that were subjected to a cleaning, crushing and sterilization process, may be an effective graft in alveolar bone defects in the same patient. Today, newly extracted teeth are used in the office to recycle them into mineralized autogenous bone from dentin for immediate grafting. This procedure is indicated in cases where teeth are removed by periodontal problems or impacted parts.

Particulate graft of mineralized dentin in extractions.

The "gold standard" for alveoli preservation, sinus membrane elevation and bone defects should be considered. When autogenous bone is used, bone reabsorption after grafting is inevitable, in addition to a second defect in the donor area.

In the alveolar bone the inorganic content is $65 \%$ and the organic content is $25 \%$. In the end $90 \%$ organic content is type I collagen that plays an outstanding role in bone formation and mineralization.

A dental part has a structure very similar to cortical bone and offers a type of graft that promotes bone formation in addition to keeping the grafted bed in optimal condition and it is proven that bone morphogenetic proteins (BMPs) promote bone morphogenetic proteins (BMPs) promote bone morphogenetic proteins (BMPs) formation of cartilage and bone and diversification of indissociable stem cells, into chondrocytes and osteogenic cells. Non-cholagen proteins such as osteocalcin, osteoconectin, phosphoprotein and sialoprotein are involved in bone calcification. Protein matrix patterns in the teeth have osteoconductive potential.

\section{Characteristics}

It is a stable short and medium term graft, it is considered as autologous graft, it is osteoinductive and osteoconductive, it is affordable as well as easy and quick to get. Healing is obtained in half the time, so rehabilitation can be done much faster. The amount of graft that can be obtained is 1 to 3 or $3.5 \mathrm{cc}$. Autogenous dentin promotes new bone growth, fuses with existing bone, forms type II bone and the time to place an implant is starting at six weeks.

\section{Process}

The preparation of the tooth consists of removing the dental tartare and the remaining periodontal tissue in the piece with a carbide cutter, if there is restoration should be removed. It is dried with oil free air, is placed in the grinder, which is Komati Bio, is programmed 3 seconds of grinding and 10 seconds of vibration to separate the large particles from the small ones, the size should be between 300 to 1, 200 microns; in case another grinding is necessary, it is carried out.

The particles are transferred to a sterile glass container, with the chemical cleaner based on sodium hydroxide and $20 \%$ ethanol, the crushing is completely covered. It should be noted that the cleaning solution is very effective for dissolving organic material, including bacteria, endotoxins and viruses. After 5 minutes, excess moisture is removed with sterile gauze. Finely pour the saline phosphate solution that will bring the pH to 7.2 and is dehydrated again with gauze, this is done twice. The graft is ready for use. It has recently been suggested to treat particulate dentin with ethyleendiamymethrostic acid (EDTA), for two minutes prior to the addition of the buffer to create a layer of only 10 microns of demineralized dentin to induce the attraction of morphogenetic protein even.

\section{Fibrin-rich plasma}

The development of an injectable (PRF) allows to find a high number of leukocytes and developmental factors, as well as allowing to bind the graft. It is a fully autologous concentrated blood system, does not require any external anticoagulant, is character- 
ized by a simplified and appropriate preparation process for use in the clinical applications of the daily journal and is made under the concept of low Spinning speed, modification of the preparation protocol is the reduction of centrifugal force, resulting in advanced PRF and an injectable fluid.

Mineralized dentin particulate matrix Compared to PRF, this new matrix is enriched with inflammatory cells such as leukocytes and platelets. In addition, the concept of low speed in the spinning, allows the development of a more porous clot in its structure to serve as a Sagrada and reservoir of growth factors that facilitates the regeneration process. When mixing the dentin particulate (and enamel) with PRF, it is suggested to dry a little more, it can be done in autoclave by a four- or five-minute program.
Results Under the scanning electron microscope Auto BT shows a consistency similar to that of cortical bone, so they can be considered to use the teeth as an autogenous graft, because they have physicochemical characteristics similar to those autogenous bone grafts. X-ray evidence shows the effectiveness of this procedure when observing bone regeneration [1-6].

\section{Conclusions}

Auto BT can be safely used within a variety of reconstruction procedures such as maxillary sinus grafting, guided bone regeneration, increased alveolar rim and graft for post-extraction alveoli, which represents a good resource since autogenous tooth grafting is safer than allogen and xenogeny grafts.

\section{Clinical cases}
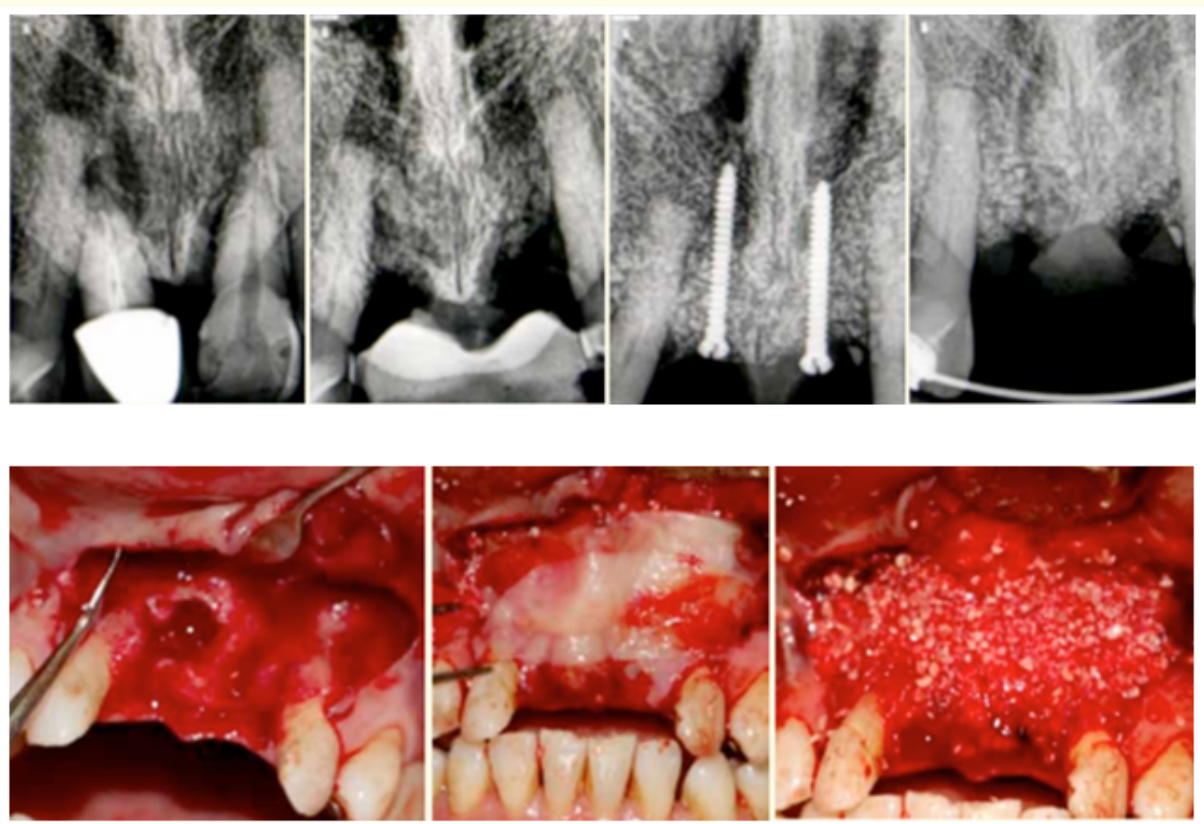

Case 1
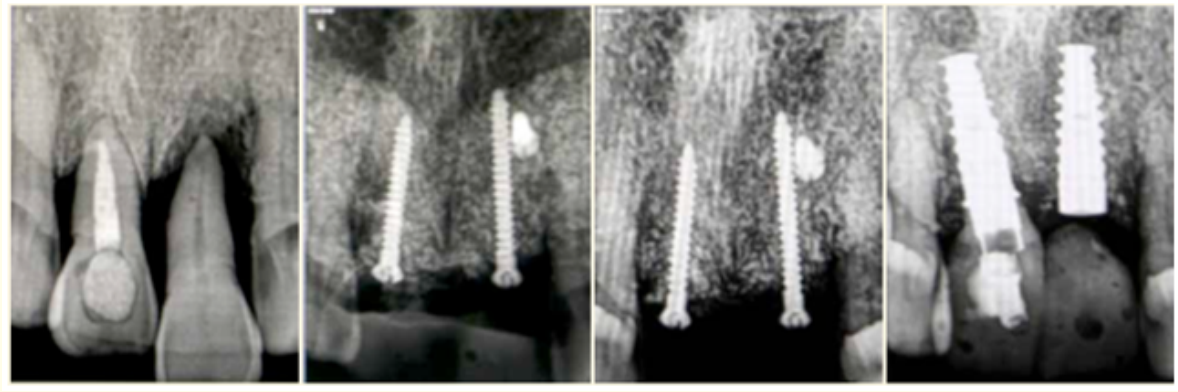

Case 2 


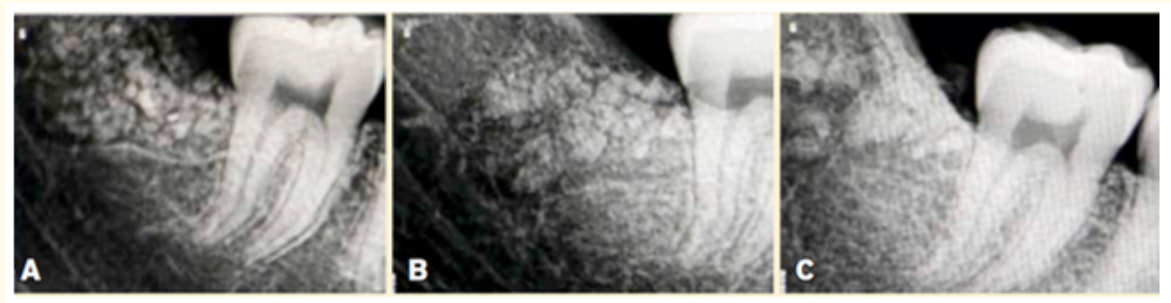

Case 3

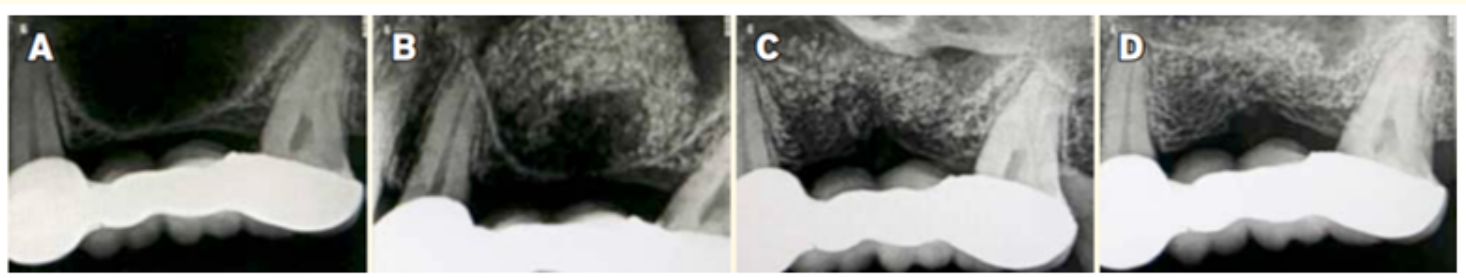

Case 4

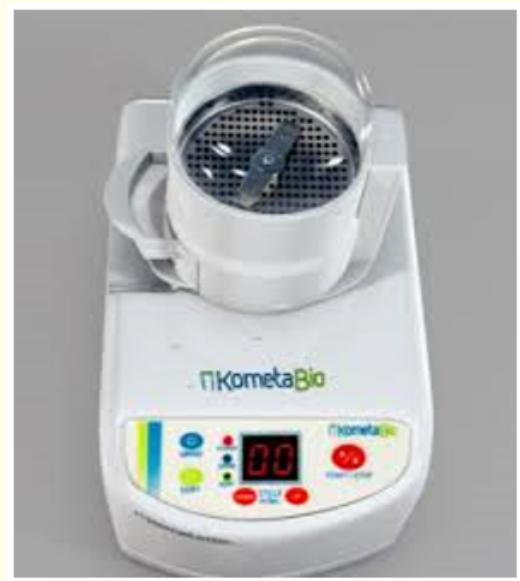

Figure 1: KometaBio, Smart Dentin Grinder.

\section{Bibliography}

1. Itzhak Binderman., et al. "A Novel Procedure to Process Extracted Teeth for Immediate Grafting of Autogenous Dentin".

2. Young Kyun Kim., et al. Autogenus teeth used for bone grafting: a comparison with traditional grafting materials; Seoul National University, Chosun University, and Kangwon National University.

3. Kyun Kim., et al. Analysis of Organic Components and Osteoconductivity in Autogenous Tooth Bone Graft Material; Young.

4. Huggins CB and Reddi AH. "Coagulation of blood plasma of guinea pig by the bone matrix". Proceedings of the National Academy of Sciences of the United States of America 70.3 (1973): 929-33.
5. T Kuboki Y., et al. "Regenerative Medicine of Bone and Teeth Kawakami”. Journal of Hard Tissue Biology 16.3 (2007): 95113.

6. Hallel G Lerreter M., et al. "FRENCH Greffe de dentine autologueet' preservation de la cre'te alveolare". L'Information Dentaire (edition 07-08-2016).

\section{Volume 3 Issue 12 December 2019}

(C) All rights are reserved by Vicente Carrasco. 